\title{
miR-23a, miR-24 and miR-27a protect differentiating ESCs from BMP4-induced apoptosis
}

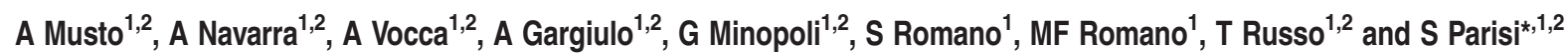

Numerous studies have indicated that BMP4 signaling is involved in the regulation of the early steps of development. In mouse embryonic stem cells (ESCs), BMP4 is crucial to sustain pluripotency and blocks differentiation towards neural fate. Here, through a systematic analysis of miRNAs in ESCs, we establish that BMP4 signaling regulates miR-23a, 27a and 24-2, through the recruitment of phospho-Smads at the promoter of the gene encoding this miRNA cluster. Suppression of $\mathrm{miR}-23 \mathrm{a} / \mathrm{b}, 27 \mathrm{a} / \mathrm{b}$ and 24 does not affect self-renewal or pluripotency, but induces an evident change of ESC differentiation, with a significant increase of the cells undergoing apoptosis after the transition from ESCs to epiblast stem cells (EpiSCs). BMP4 has been previously reported to cause apoptosis during ESC differentiation. By blocking BMP4 signaling, we completely prevent the apoptosis induced by suppression of the miRs. This suggests that the effects of miR suppression are the result of enhanced BMP4 signaling. This hypothesis is further supported by the observation that Smad5, the transcription factor downstream of the BMP4 receptor, is targeted by the miRNAs of the 23a and 23b clusters. Altogether, our results highlight the existence of a regulatory loop, involving Smad5 and the miR-23a clusters, that modulates the apoptotic response of ESCs to BMP4.

Cell Death and Differentiation (2015) 22, 1047-1057; doi:10.1038/cdd.2014.198; published online 5 December 2014

ESCs represent a precious experimental model of the early stages of embryo development. They can be grown indefinitely in vitro and induced to differentiate, thus mimicking two events taking place in the blastocyst: (i) the differentiation of the cells of the inner cell mass into epiblast cells and (ii) the commitment of epiblast cells to neuroectodermal or mesendodermal precursors. ${ }^{1,2}$ These differentiation events are regulated by extrinsic signals. BMP4, a member of the TGF$\beta$ superfamily of cytokines, has a crucial role in ESCs. Many results indicate that it is able, together with LIF, to maintain mouse ESCs in the pluripotent state. ${ }^{3}$ This effect is mediated by the regulation of many direct targets of Smad1, 5 and 8, the transcription factors downstream of the BMP4 receptor. ${ }^{4}$

BMP4 also contributes to govern early steps of differentiation. First, BMP4 acts by regulating ESC-epiblast transition and then by suppressing neural differentiation and promoting non-neural lineage formation. ${ }^{2}$ Moreover, BMP4 promotes the differentiation towards mesendodermal lineages and also regulates, either positively or negatively, the further commitment of mesendodermal precursors into their different fates. $^{5-8}$

Gene expression in ESCs is regulated by a complex network of transcription factors..$^{9,10}$ In addition, numerous results indicate that miRNAs are crucial regulators of the gene expression programs that drive ESC differentiation. Suppression of miRNA biogenesis, obtained by knocking out Dicer or
DCGR8 genes, leads to an early arrest of mouse embryonic development and of in vitro differentiation of ESCs. ${ }^{11,12}$ The most abundant miRNAs in ESCs belong to the mouse miR-290 family and to their human counterpart miR-302 family. ${ }^{13,14}$ These miRs are mainly involved in cell cycle regulation and in the prevention of the epigenetic silencing of pluripotent factors, like Oct3/4, Sox2, Nanog and Myc. ${ }^{15,16}$ Many other miRNAs, not specific of ESCs, have been found to regulate important steps of ESC differentiation, both in vitro and in vivo. ${ }^{17}$

We have studied the expression profiles of miRNAs during mouse ESC differentiation. ${ }^{18}$ Among these miRs, we found that miR-125a regulates the fine balance between BMP4 and Nodal/Activin pathways in the initial phases of ESC differentiation. ${ }^{19}$ This regulation occurs through an efficient auto-regulatory loop in which BMP4 controls the transcription of miR-125a that targets the BMP4 co-receptor, Dies $1 .{ }^{20}$ As a consequence, this mechanism sets ESC sensitivity to BMP4. It is quite reasonable that the interplay between the BMP4 pathway and the transcription of miRNAs may represent a more general regulatory mechanism modulating the response of ESCs to extracellular stimuli. Therefore, we have analyzed the miRNAs regulated by BMP4 in ESCs. We found that the $\mathrm{miR}-23 \mathrm{a} / 24-2 / 27 \mathrm{a}$ cluster is regulated by BMP4 at transcriptional level. These miRNAs are highly expressed in ESCs and are essential to protect these cells from apoptosis during

\footnotetext{
'Department of Molecular Medicine and Medical Biotechnology, University of Naples 'Federico Il', Via Sergio Pansini 5, Naples, Italy and ${ }^{2}$ Ceinge Biotecnologie Avanzate, Via Gaetano Salvatore 486, Naples, Italy

*Corresponding author: S Parisi, Department of Molecular Medicine and Medical Biotechnology, University of Naples, 'Federico Il', Via Sergio Pansini 5, Naples 80131, Italy. Tel: +39 081373 7878; Fax: +39 081373 7808; E-mail: silvia.parisi@unina.it

Abbreviations: ESCs, embryonic stem cells; EpiSCs, epiblast stem cells; miRs, microRNAs; UTR, untraslated region; pri-miRNA, primary transcript of miRNA; SFEBs, serum-free embryoid bodies; BMP4, bone morphogenetic protein; DCGR8, DiGeorge syndrome critical region 8; Dies1, differentiation of embryonic stem cells 1; ChIP, chromatin immunoprecipitation; qPCR, quantitative PCR; KSR, knock-out serum replacement; Id, inhibitor of differentiation; TGF $\beta$, transforming growth factor beta; PI, propidium iodide; KD, knock-down.

Received 02.6.14; revised 17.9.14; accepted 01.10.14; Edited by M Piacentini; published online 05.12.14
} 
differentiation. This function is fulfilled through the regulation of Smad5 level, a direct target of these miRNAs. Moreover, we have demonstrated that these miRNAs act by modulating the strength of BMP4 signaling in differentiating ESCs and that a slight alteration of BMP4 signaling, due to miRNA suppression, results in an increase of the physiological apoptosis that occurs in differentiating ESCs.

\section{Results}

miR-23a, miR-24-2 and miR-27a are regulated by BMP4. To identify the miRNAs whose transcription is under the control of BMP4, we exposed ESCs to BMP4 in a medium containing Knock-out Serum Replacement plus LIF to avoid the influences of growth factors contained in the serum and to switch-off the exogenous TGF $\beta$ signaling. We assessed that BMP4 signaling was activated by measuring the induction of Smad target genes, $I d 1$ and $I d 3$, the accumulation of mature miR-125a at $24 \mathrm{~h}$ and Smad phosphorylation (Supplementary Figure S1A-C). ${ }^{19,21}$ The time point of sample collection was fixed at $24 \mathrm{~h}$ of BMP4 treatment to allow completion of the processing of all miRNA primary transcripts (pri-miRNA). The level of single miRNAs was analyzed by using a miRNA qPCR panel. After selection for S.E. and fold change, we clustered the miRNAs in two groups on the basis of their behavior on BMP4 treatment (Supplementary Table S1). Of note, we found the miR-125a upregulated by BMP4, in agreement with our previous observations. ${ }^{19}$ In addition, to select miRNA genes directly regulated by BMP4 signaling, we measured the level of pri-miR transcripts after $1 \mathrm{~h}$ of BMP4 and we found a significant change in the levels of the pri-miR transcript for miR-23a, miR-24-2 and miR-27a (Figure 1a). These miRNAs are encoded by the same cluster gene on the chromosome 8 (miR-23a cluster). Q-PCR analysis of these miRs confirmed their increase on BMP4 treatment (Figure 1b). We also observed the decrease of the primary transcript of the miR-23a cluster on inhibition of BMP4 signaling with BMP4 antagonist Dorsomorphin (Figure 1c). Of note, also the silencing of Smad1 and Smad5 by RNAi (Supplementary Figure S1D) resulted in a marked decrease of the miR-23a cluster primary transcript (Figure 1d). To demonstrate that miR-23a cluster is controlled by the BMP4 pathway directly at a transcriptional level, we assessed Smad binding on the promoter of miR-23a cluster gene using chromatin IP (ChIP). As shown in Figure 1e, on BMP4 treatment, activated Smads bind to Id1 promoter ${ }^{3}$ and at the same time we found enrichments in the chromatin immunoprecipitated with an anti-Smad antibody in the region upstream of the transcriptional start site of the miR-23a cluster gene (Figure 1f). Accordingly, sequence analysis of the genomic region upstream the miR-cluster identified many consensus binding sites for Smads. ${ }^{4}$ These data indicated that transcription of the miR-23a cluster gene is regulated by BMP4.

Suppression of miRNAs of the $23 a$ and $23 b$ clusters affects ESC differentiation. All the miRNAs of the $23 a$ cluster are expressed in undifferentiated ESCs and their levels decrease soon after differentiation induction, although they are still significantly expressed during differentiation until the formation of neuroectodermal cells (Supplementary Figure S2A). Another miRNA cluster, located on chromosome 13, contains miR-23b, miR-24-1 and miR-27b; miR-24-1 is identical to miR-24-2 and miR-23b and 27b belong to the same families of miR-23a and $27 a$ and share the same putative targets. miR-23b and $27 b$ as well as the primary transcript of the miR-23b cluster are expressed in ESCs, decrease during differentiation and only their mature forms are induced on BMP4 treatment (Supplementary Figure S2B-D).

We studied the function of these miRNAs by inhibiting them with specific anti-miRs (Supplementary Figure S2E) during ESC differentiation through the formation of serum-free embryoid bodies (SFEBs) that result in the generation of epiblast stem cells (EpiSCs) before day 2, that then differentiate into neuroectodermal precursors ${ }^{2,19}$ before day 4. First, we analyzed the possible effect of suppression of all miRNAs of the $23 a$ and $23 b$ clusters on ESC selfrenewal. As shown in Figure $2 a$, we did not find any significant changes in the expression of stemness markers on miRNA suppression. Then, we analyzed the effects of the suppression of miR-clusters on ESC differentiation. At 4 days of differentiation, SFEBs are composed mainly of neuroectodermal cells (Sox1-positive cells) and of some pluripotent cells (Oct3/4positive cells) present within the embryoid bodies. While no significant differences were found in neuroectodermal markers (Sox1 and Pax6), the expression of Oct3/4 and Nanog was greatly decreased in the SFEBs where the miRNAs were suppressed (Figure 2b). It is worth noting that these changes were not observed on suppression of the single miRNAs (Supplementary Figure S2F,G) and that the rapid decrease of stemness markers observed on miRNA cluster suppression was not due to an altered proliferation rate of pluripotent stem cells, as measured by BrdU incorporation (Supplementary Figure S3A).

miRNAs of the 23a and 23b clusters suppress apoptosis during ESC differentiation. To examine in detail the relative composition of pluripotent stem cells and differentiated cells within the SFEBs, we analyzed sectioned SFEBs by immunostaining, on the suppression of the miRNAs of 23a and $23 \mathrm{~b}$ clusters. We observed that control SFEBs contain clumps of Oct3/4-positive cells surrounded by neuroectodermal cells (Sox1-positive) at 4 days of differentiation. On the contrary, SFEBs derived from miRNA KD ESCs showed an evident reduction or even absence of Oct3/4-positive cells, with extensive cell death in the center of the embryoid bodies (Figures 2c and d). To determine whether the decrease of stem cells during differentiation was related to increased apoptosis, we analyzed the positivity to an antibody directed against cleaved caspase-3. As shown in Figure 3a, during ESC differentiation a relatively small number of cells spontaneously undergoes apoptosis, while about $10 \%$ of SFEBs derived from ESCs transfected with inhibitors of miRclusters show large areas of positivity to active caspase-3, mainly localized in the center of the SFEBs and surrounded by Sox1-positive cells (Figure 3a). The effect on apoptosis due to suppression of miR-23a and $23 \mathrm{~b}$ clusters was also supported by the increased level of cleaved PARP 
a
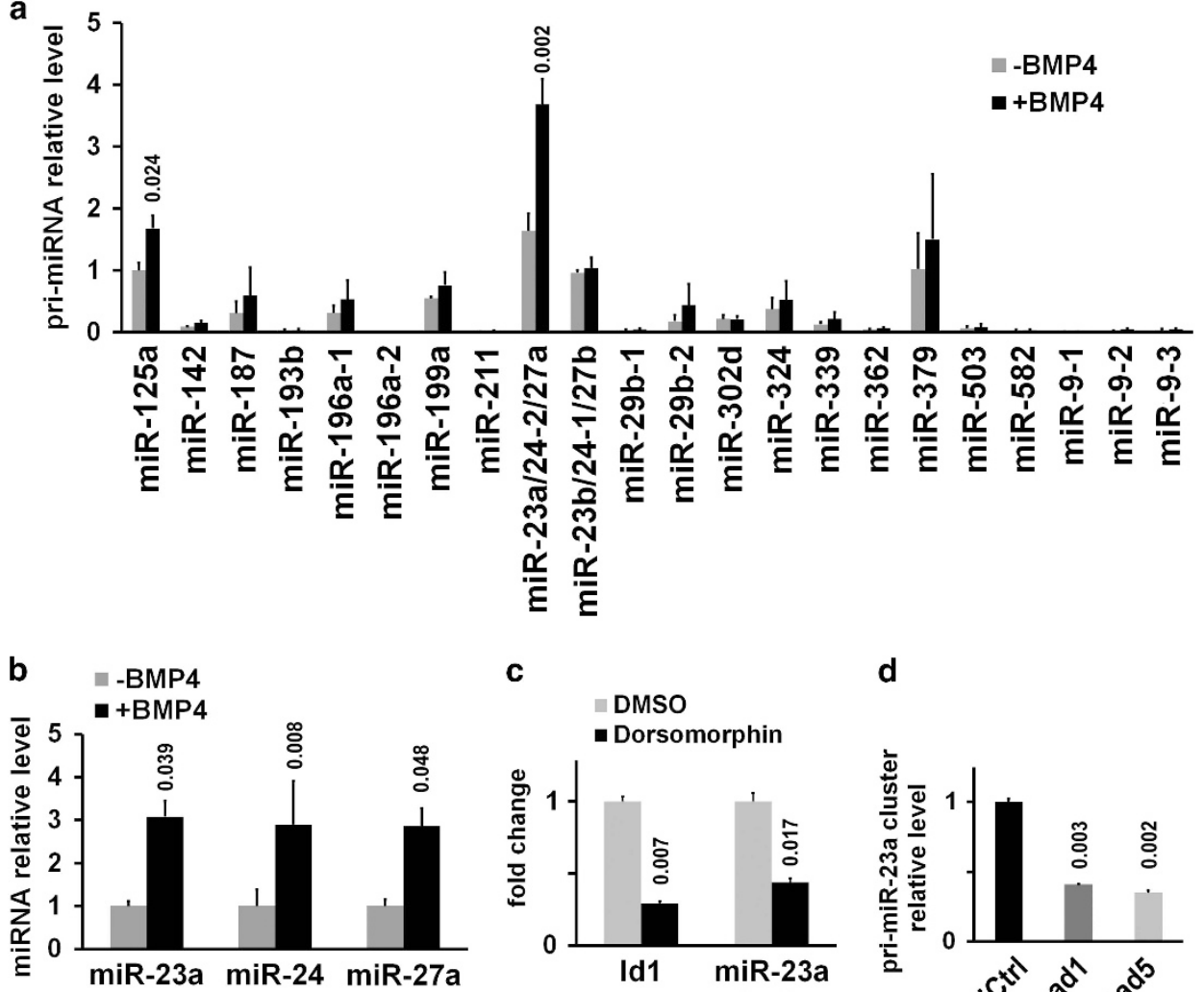

C

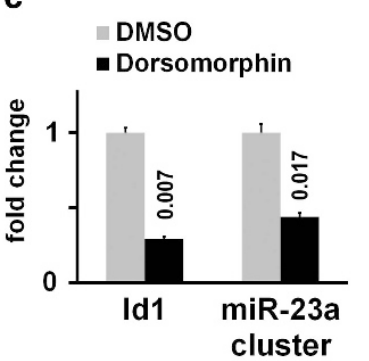

d

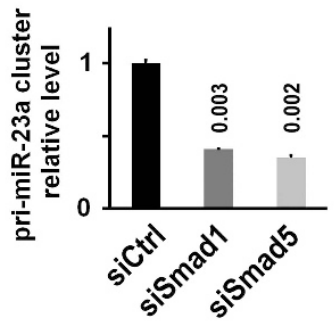

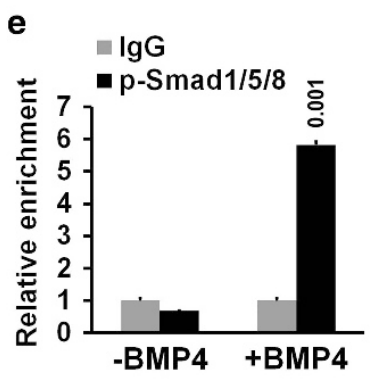

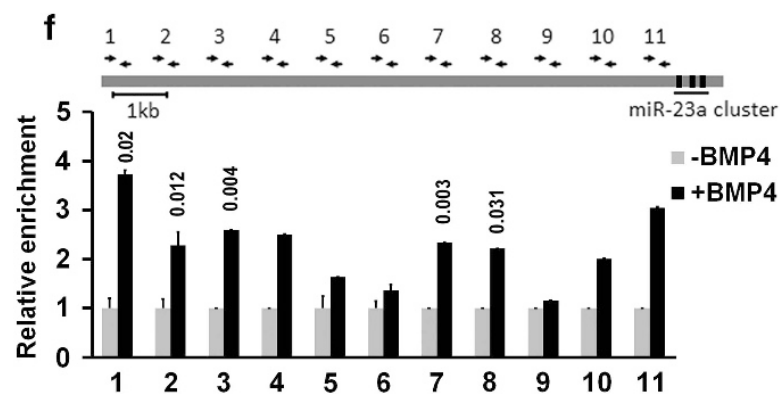

Figure 1 Regulation of miR-23a cluster by BMP4 signaling. (a) ESCs were cultured overnight in chemically defined medium (KSR) with LIF and treated with $20 \mathrm{ng} / \mathrm{ml}$ of BMP4 for $1 \mathrm{~h}$. Expression level of the primary transcripts of upregulated miRNAs was analyzed by qPCR. Data are presented as fold change over the level of the miR-125a in untreated (-BMP4) ESCs (fold change $=1$ ) $(\mathbf{b})$ Accumulation of mature miRNAs was analyzed by qPCR in ESCs after $24 \mathrm{~h}$ of BMP4 treatment. Data are presented as fold change over the level of the indicated miRNA in untreated $(-B M P 4)$ ESCs (fold change $=1)(c)$ The level of BMP4 target gene Id1 and the primary transcript of miR-23a cluster was analyzed by qPCR on signaling inhibition by Dorsomorphin treatment for $1 \mathrm{~h}$. Data are expressed as fold change relative to control (DMSO) ESCs (fold change $=1$ ) (d) ESCs were transfected with the indicated siRNAs and the level of the primary transcript of miR-23a cluster was measured by qPCR after $24 \mathrm{~h}$. Data are expressed as fold change relative to the levels of control (siCtrl) cells (fold change $=1$ ) $(\mathbf{e})$ Phospho-Smad1/5/8 binding to the promoter region of Id1 was analyzed by ChIP-qPCR after $1 \mathrm{~h}$ of BMP4 treatment compared with untreated cells. Data are expressed as fold enrichment relative to the IgG controls (fold change $=1$ ) (f) Phospho-Smad association with the genomic upstream region of the transcription start site of the miR-23a cluster gene. ESCs were treated or not with BMP4 and the samples were subjected to ChIP with the antibody for p-Smad1/5/8. Immunoprecipitated DNA was analyzed by qPCR with primers amplifying specific promoter regions indicated by the arrows in the top panel. Data are presented as fold enrichment relative to the untreated $(-B M P 4)$ cells (fold change $=1)$. $(N \geqslant 3)$. The numbers over the bars indicate the $P$-Value

(Figure 3b). Moreover, FACS analysis highlighted that the percentage of hypodiploid cells is significantly increased in differentiating cells on miRNA suppression (Figure 3c). To understand when the apoptosis was triggered, we analyzed the pattern of caspase-3 activation at different time points. As shown in Supplementary Figure S3B, we observed that the suppression of the two clusters does not affect apoptosis in undifferentiated cells.
Then, to understand when apoptosis starts during differentiation, we first analyzed the transition from ESCs to EpiSCs. ${ }^{22,23}$ As shown in Figure $4 a$, formation of EpiSCs is not impaired by suppression of miR-clusters as indicated by the increase of the epiblast markers (Fgf5, Otx2 and Dnmt3b), the decrease of the ESC-specific marker Dax1 and the maintenance of Nanog. Analysis of active caspase-3 shows that suppression of miR-clusters does not induce abnormal 
a

$$
\begin{aligned}
& \text { anti-miR-ctrl } \\
& \text { - anti-miR-23a/b clusters }
\end{aligned}
$$

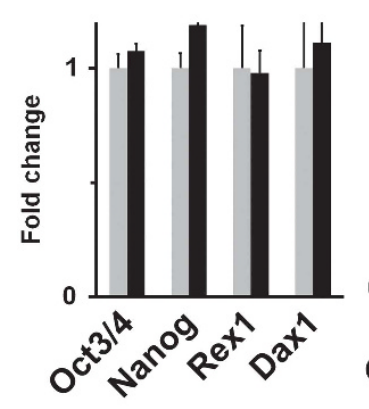

b

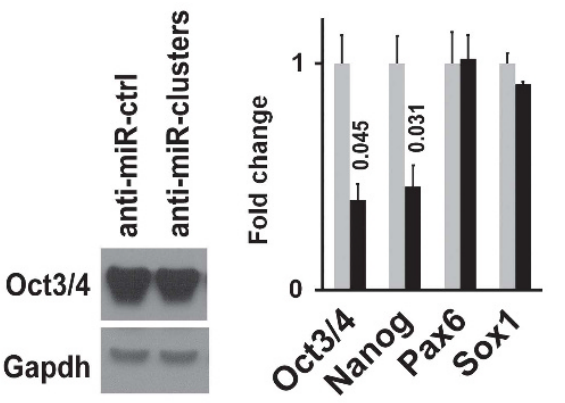

- anti-miR-ctrl - anti-miR-clusters

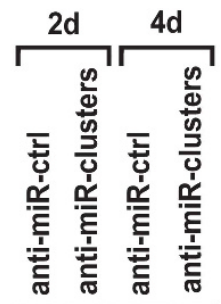

Oct3/4

Nanog

Gapdh

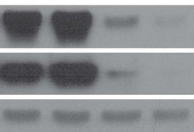

Pax6

Sox 1

Gapdh

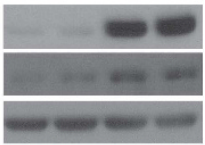

C
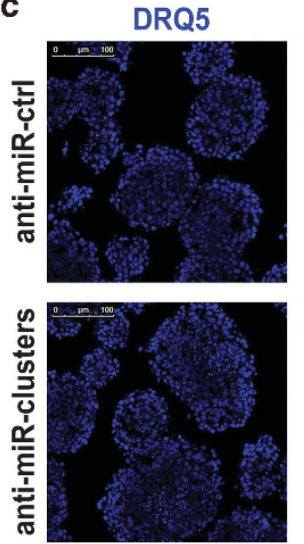

Oct3/4/Sox 1
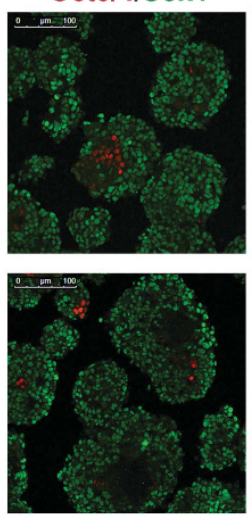

d
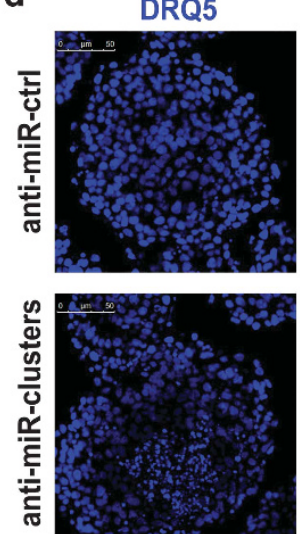

Oct $3 / 4 /$ Sox 1
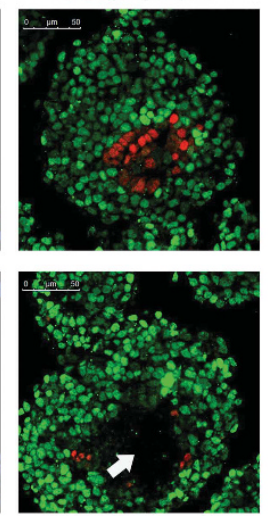

Figure 2 Effects of miRNA suppression on ESC self-renewal and differentiation. (a) Undifferentiated ESCs were transfected with specific anti-miRs for miRNAs of 23a and $23 \mathrm{~b}$ clusters (anti-miR-clusters) or with a control anti-miR (ctrl) and after $24 \mathrm{~h}$ the expression of stemness markers was analyzed by qPCR (left panel) and western blot (right panel). Data are expressed as fold change relative to control (anti-miR-ctrl) ESCs (fold change $=1$ ). (b) ESCs were induced to differentiate through SFEB formation on suppression of the miRNAs of 23a and 23b clusters. Levels of stemness (Oct3/4, Nanog) and neuroectodermal markers (Pax6, Sox1) were analyzed by qPCR (at 4 days of differentiation) and western blot (at 2 and 4 days of differentiation). Data are expressed as fold change relative to control (anti-miR-ctrl) SFEBs (fold change $=1$ ). The numbers over the bars indicate the P-Value. (c) ESCs transfected with the indicated anti-miRs were induced to differentiate through SFEB formation for 4 days and then subjected to immunostaining to analyze the expression of stemness (Oct3/4) and neuroectodermal markers (Sox1). Scale bars: $100 \mu \mathrm{m}$. (d) Magnification of representative SFEBs obtained from ESCs transfected with the indicated anti-miRs after 4 days of differentiation. The presence of apoptotic cells in the center of SFEBs is indicated by the white arrow. Scale bars $50 \mu \mathrm{m}$

apoptosis during epiblast transition (Figure 4b). These results suggest that apoptosis starts after ESC-EpiSC transition (0-2 days). Thus, we analyzed the onset of apoptosis at 2 and 3 days of SFEB differentiation, when epiblast transition has already occurred. Western blot analysis for cleaved caspase-3 shows that differentiation is accompanied by a significant increase of apoptosis on suppression of miR-clusters already at 2 days of differentiation (Figure 4c). Immunostaining analysis for active caspase- 3 confirmed the presence of clumps of apoptotic cells at 2 days of differentiation with a further increase in the next 2 days (Figure 4d). To further prove the role of these miRNAs in the survival of ESCs during differentiation, we have used a methods that drives an almost homogenous differentiation of ESCs towards neuroectodermal fate. ${ }^{24}$ We have employed a reporter ESC cell line expressing GFP under the control of Sox1 promoter that is specific of neural precursors (Sox1-GFP cells). ${ }^{25}$ We have found that, on suppression of miRNAs of the $23 \mathrm{a}$ and $23 \mathrm{~b}$ clusters, a significant percentage of Sox1-GFP-positive cells undergo apoptosis (Figure 4e). All these results suggest that the proper levels of miRNAs of these two clusters is fundamental to control the balance between survival and apoptosis during differentiation.

Although the basal apoptosis rate of undifferentiated ESCs is almost undetectable (Supplementary Figure S3B), these cells are highly sensitive to DNA damage..$^{26-28}$ To check whether these miRs generally protect ESCs from apoptosis, we exposed ESCs transfected with anti-miRs to 5 Gy of $X$ rays. In these conditions we observed increased apoptosis, as demonstrated by the accumulation of active caspase-3 (Figure 4f).

Taken together these results indicate that miR-clusters act to balance apoptosis versus survival of ESCs during neuroectodermal differentiation and have anti-apoptotic function in undifferentiated cells. 
a

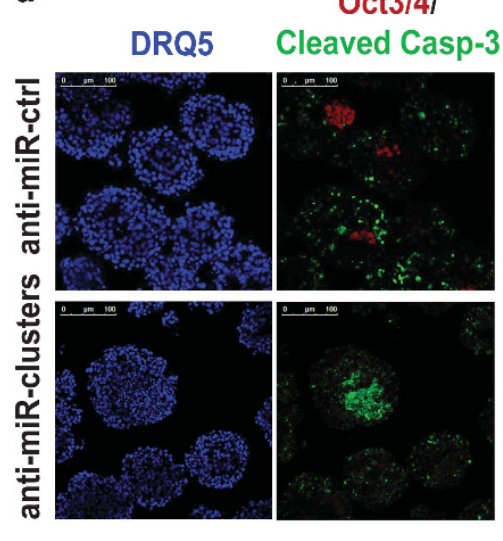

Oct $3 / 4 I$

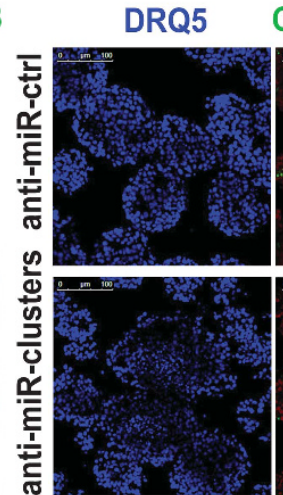

Sox1/ Cleaved Casp=3
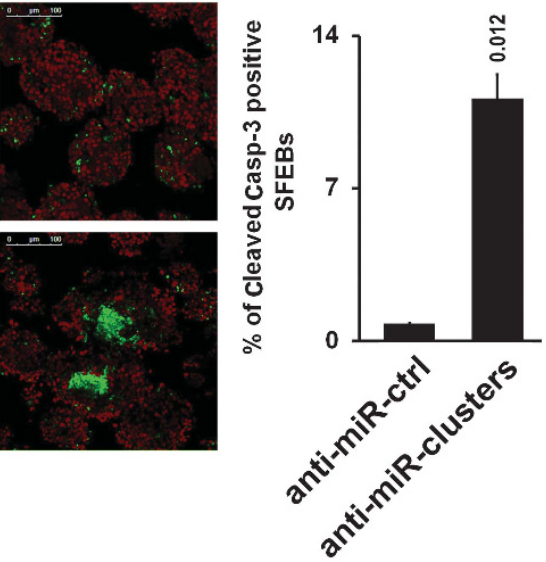

b

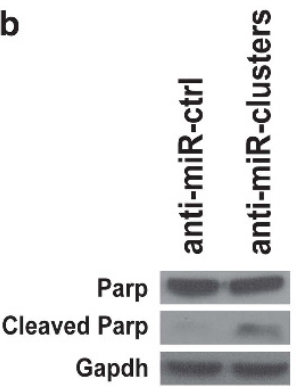

C

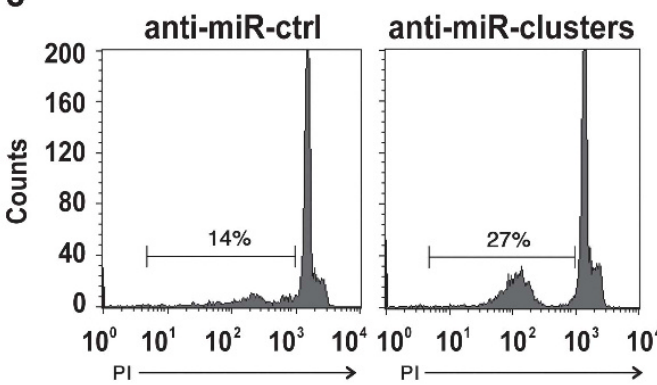

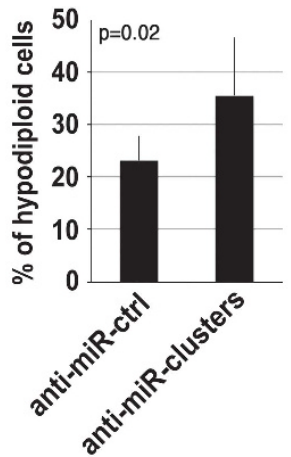

Figure 3 Apoptotic phenotype induced by suppression of the miRNAs of 23a and 23b clusters during ESC differentiation. (a) The presence of apoptotic cells within SFEBs derived from ESCs transfected with the indicated anti-miRs was analyzed by immunostaining for cleaved caspase-3 and for markers of pluripotent (Oct3/4) and neuroectodermal cells (Sox1). Scale bars: $100 \mu \mathrm{m}$. The graph reports the percentage of SFEBs showing an extensive apoptotic area. (b) The levels of cleaved PARP were measured by western blot analysis in SFEBs derived from ESCs transfected with the indicated anti-miRs. (c) PI staining of SFEBs at 4 days of differentiation derived from ESCs transfected with anti-miR-ctrl or anti-miR-clusters. The percentage of hypodiploid PI-positive cells measured by FACS analysis is reported $(n=6)$

Suppression of miRNAs of the $23 a$ and $23 b$ clusters enhances intracellular signaling downstream of BMP4. We have observed that suppression of miRNAs of the 23a and $23 \mathrm{~b}$ clusters results in evident apoptosis during ESC differentiation toward neuroectoderm. It has been demonstrated that treatment of differentiating ESCs into SFEBs with high doses of BMP4 $(10 \mathrm{ng} / \mathrm{ml})$ blocks differentiation thus maintaining pluripotency. ${ }^{2}$ In contrast, it was also observed that low doses of BMP4 induce apoptosis during epidermal differentiation of ESCs. ${ }^{29}$ We reasoned that suppression of miR-clusters can result in an enhancement of the endogenous signaling that mimics the exposure of the cells to low doses of BMP4. Thus, we treated ESCs with different doses of BMP4 and the onset of apoptotic phenotype was analyzed by measuring the level of cleaved caspase-3. We found that treatment with low doses of BMP4 results in an evident increase of active caspase-3, whereas high dose of BMP4 does not trigger apoptosis but leads, as expected, to block ESC differentiation (Figure $5 \mathrm{a}$ and Supplementary Figure S4A). Then, we treated differentiating ESCs, transfected with anti-miR-clusters, with the BMP4 antagonist Dorsomorphin. The rationale of this experiment was that if miRNA suppression leads to a reinforcement of BMP4 signaling, block of the receptor should prevent this effect and revert the apoptotic phenotype. As shown in Figure 5b, we found that the level of active caspase-3 in SFEBs derived from ESCs transfected with anti-miR-clusters and treated with Dorsomorphin are comparable to that of the control. Immunostaining analysis confirmed that accumulation of apoptotic cells in the center of SFEBs on suppression of the miR-clusters drastically decreased following Dorsomorphin treatment (Figure 5c and Supplementary Figure S4B). Similar results were found when we induced the differentiation of Sox1-GFP cells through monolayer method ${ }^{25}$ into neuronal precursors on suppression of the miRNA clusters in presence or not of Dorsomorphin (Supplementary Figure S4C). Moreover, to further demonstrate that these miRNAs mediate the BMP4-induced apoptosis we blocked the signaling at intracellular level by silencing Smad1 and Smad5, the two BMP4 effectors highly expressed in ESCs as reported below. We found that the contemporary silencing of the miRNAs and of Smad leads to a reversion of the apoptotic phenotype (Supplementary Figure S5A). These results indicated that the block of the BMP4 signaling both at membrane and at 
a

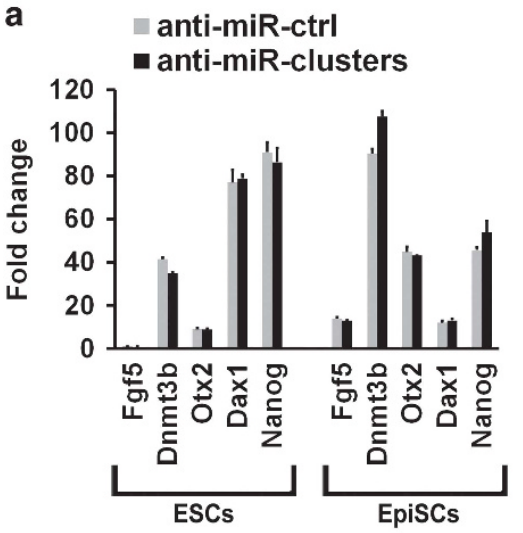

b

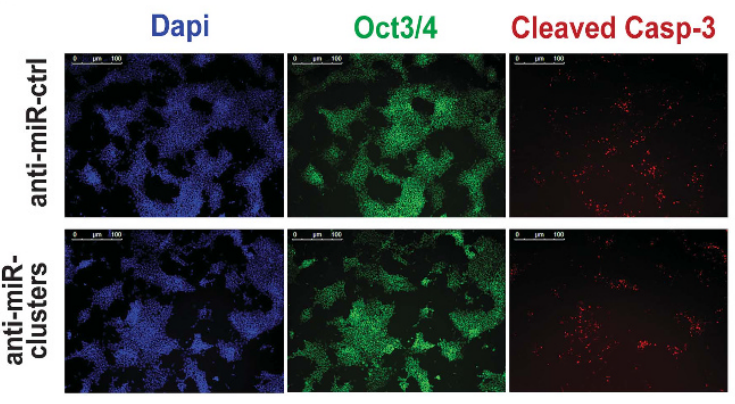

C

d

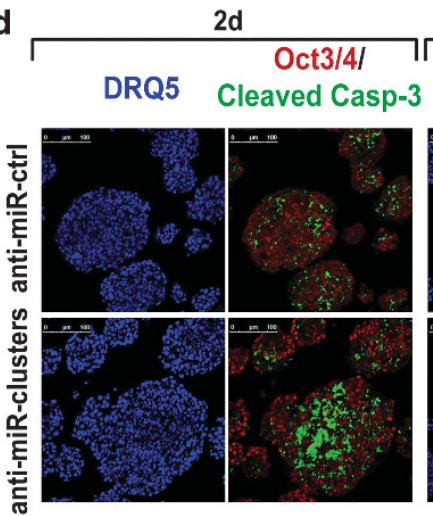

$2 d$

$3 d$

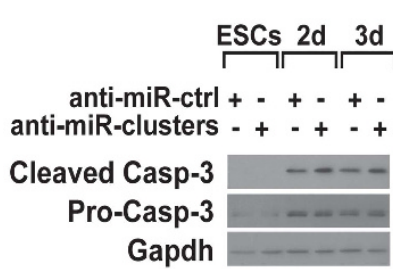

e
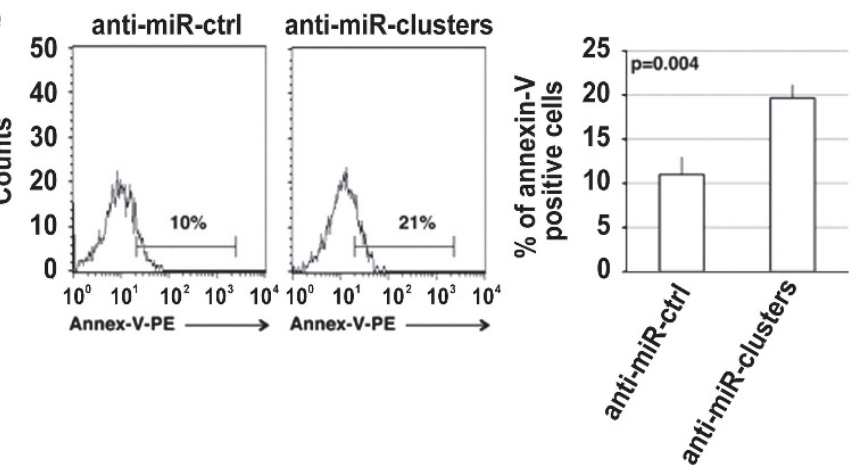

f DRQ5 Cleaved Casp-3
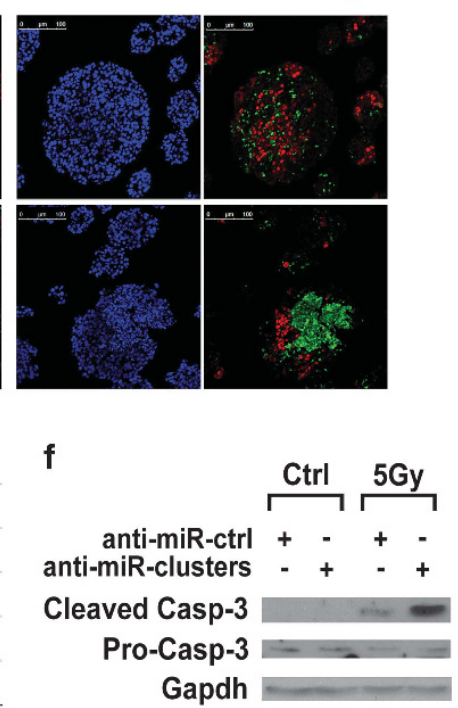

Figure 4 Apoptosis induced by the suppression of the miRNAs of the 23a and 23b clusters occurs after ESC-EpiSC transition. (a) ESCs were transfected with the indicated anti-miRs and were induced to differentiate into EpiSCs. Markers of ESCs (Nanog and Dax1) and EpiSCs (Otx2, Dnmt3b, Fgf5, Nanog) were measured by real-time PCR in both conditions. Data are presented as fold change over the level of the Fgf5 mRNA in ctrl (anti-miR-ctrl) ESCs (fold change $=1$ ). (b) The presence of apoptotic cells in EpiSCs after suppression of the miRNAs of 23a and 23b clusters was analyzed by immunostaining with an anti-cleaved caspase-3 antibody. Scale bars: $100 \mu \mathrm{m}$. (c) ESCs were transfected with the indicated anti-miRs and the level of cleaved caspase-3 was measured by western blot in undifferentiated cells and at 2 and 3 days of SFEB differentiation. (d) The presence of apoptotic cells and pluripotent stem cells in SFEBs was analyzed by immunostaining at 2 and 3 days of differentiation. Scale bars: $100 \mu \mathrm{m}$. (e) Annexin-V staining of Sox1-GFP ESCs transfected with anti-miR-ctrl or anti-miR-clusters and differentiated for 4 days in monolayers to obtain the conversion into neuroectodermal cells. ${ }^{24}$ The percentage of Annexin-V-positive cells measured by FACS analysis is reported $(n=3)$. (f) ESCs transfected with the indicated anti-miRs were irradiated ( 5 Gy) or not (ctrl) with $X$ rays and after $24 \mathrm{~h}$ the levels of cleaved caspase- 3 were evaluated by western blot

intracellular levels is able to rescue the protective effect of miRNAs of $23 \mathrm{a}$ and $23 \mathrm{~b}$ clusters against the apoptosis triggered by low BMP4 signaling.

A strong support to the hypothesis that suppression of miRNAs of $23 \mathrm{a}$ and $23 \mathrm{~b}$ clusters is reinforcing the intracellular signaling downstream of BMP4 comes from the analysis of the targets of these miRNAs. We found that among the many predicted targets (Targetscan and miRWalk), the miRNAs of $23 \mathrm{a}$ and $23 \mathrm{~b}$ clusters could target several Smads, the canonical effectors of BMP4 signaling. Moreover, Rogler et $a l^{30}$ have demonstrated that the miRNAs of 23b cluster target Smad3, 4 and 5 in liver stem cells. ${ }^{29}$ We first analyzed the expression profiles of these Smads in ESCs and during differentiation. We found that Smad3 is almost undetectable in ESCs and in the first days of differentiation. Instead, Smad4 and 5 are expressed in ESCs and significantly increase during differentiation, when the expression of miR-clusters decreases (Figure $6 \mathrm{a}$ and Supplementary Figure S2). 
a

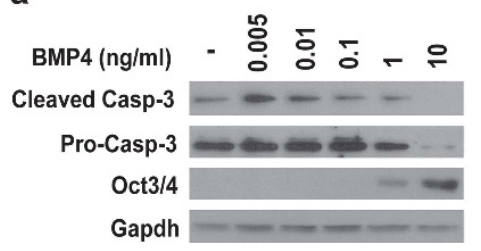

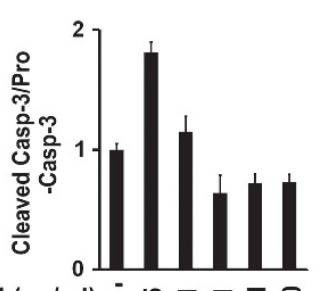

BMP4 (ng/ml) ' b $\mathrm{DMSO}++\cdots$

Dorso. - - + +

anti-miR-ctrl $+\cdot+\cdot$

anti-miR-clusters - + + +

Cleaved Casp-3 $\approx-\infty$

Pro-Casp-3 ---

Gapdh

C

anti-miR-ctrl
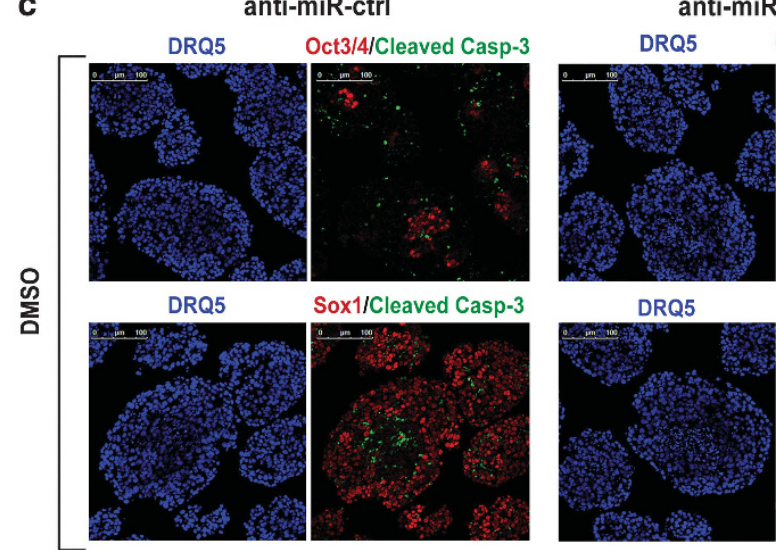

anti-miR-clusters

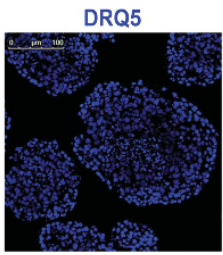

Oct3/4/Cleaved Casp-3
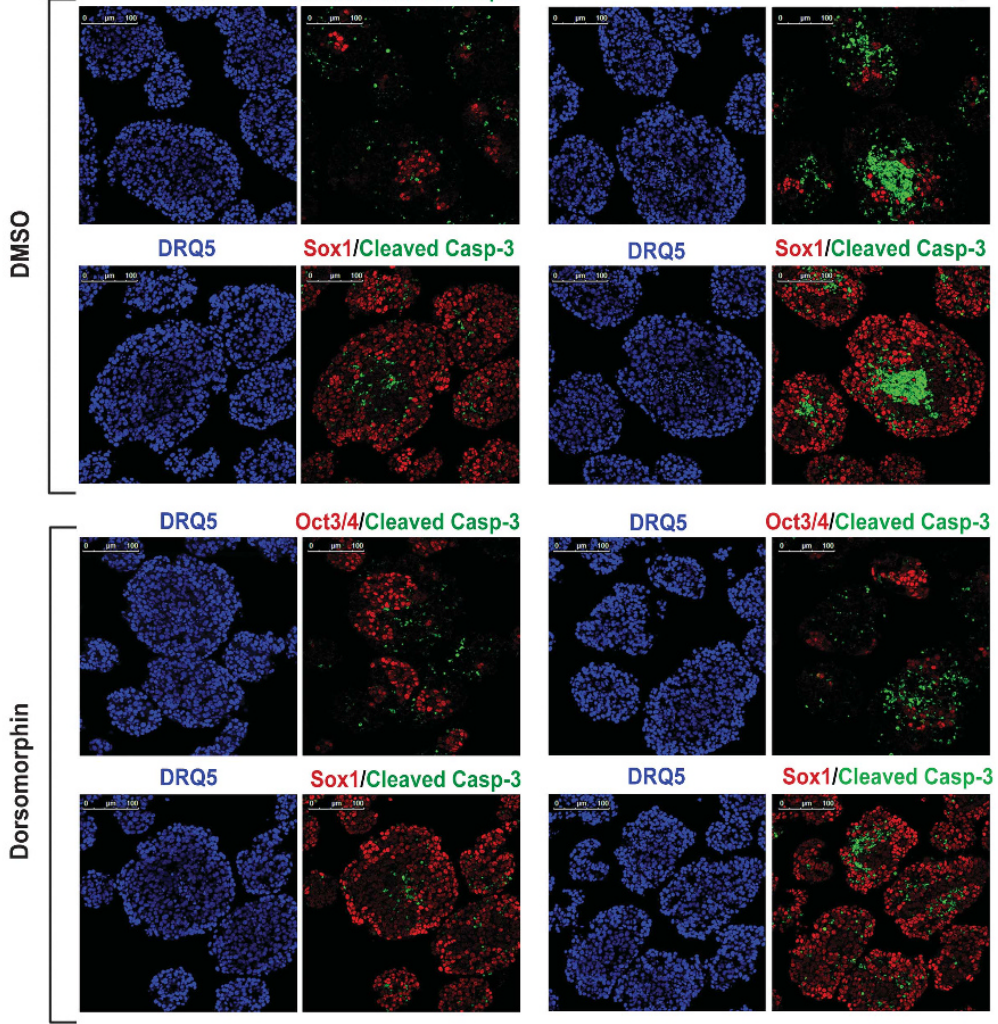

Sox1/Cleaved Casp-3

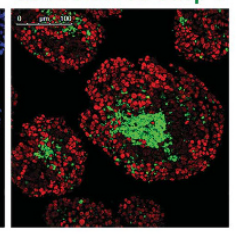

Oct3/4/Cleaved Casp-3
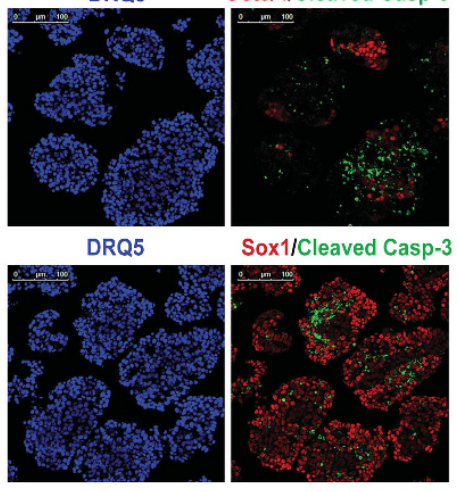

Sox1/Cleaved Casp-3

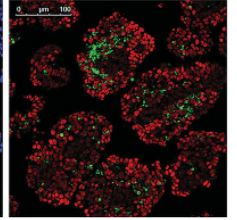

Figure 5 Apoptosis induced by the suppression of the miRNAs of 23a and 23b clusters is associated with a reinforcement of BMP4 signaling. (a) The apoptotic effect of different doses of BMP4 was analyzed by measuring the level of cleaved caspase-3 in SFEBs derived from ESCs transfected with the indicated anti-miRs and treated with the indicated amount of BMP4. Note that high doses $(10 \mathrm{ng} / \mathrm{ml}$ ) of BMP4 blocks differentiation (as demonstrated by the high levels of Oct3/4) and this results in a reduction of the levels of pro-caspase-3. The histogram reports the ratio of cleaved caspase-3/pro-caspase-3 by densitometric analysis. (b) ESCs transfected with the indicated anti-miRs were induced to differentiate through SFEBs in the presence of the BMP4 inhibitor Dorsomorphin or DMSO as control. At day 4 of differentiation protein extracts of SFEBs were analyzed by western blot. (c) Rescue of the apoptotic phenotype on Dorsomorphin treatment (lower panel) in SFEBs was analyzed by immunostaining with an anti-cleaved caspase-3 antibody. The presence of pluripotent stem cells and neuroectodermal cells was analyzed with anti-Oct3/4 and with Sox 1 antibodies, respectively. Scale bars: $100 \mu \mathrm{m}$

To understand whether expression of Smad4 and 5 can be regulated by miRNAs of $23 a$ and 23 b clusters, we knocked down the miRNAs and analyzed Smad protein levels. We found that Smad5 significantly accumulated on miRNA suppression, whereas we did not find any alterations of Smad4 protein levels (Figure 6b), indicating that Smad5 is a direct target of the miRNAs of $23 a$ and $23 b$ clusters in ESCs.

Thus, all these results indicate that BMP4 controls the level of miR-23a cluster that in turn negatively modulates the expression of Smad5, and this loop could control the balance between survival and apoptosis during ESC differentiation. On the basis of this idea we guessed that the increase of miR-23a cluster by BMP4 signaling can occur only when this signaling is strong enough to drive its transcriptional activation. To test this hypothesis we measured the level of pri-miR-23a cluster on treatment of BMP4 at low and high doses. As shown in Figure $6 \mathrm{c}$, we found that transcription of this cluster is efficiently improved only at doses of BMP4 high enough to induce the block of differentiation rather than to trigger apoptosis. The behavior of Smad5 confirmed the existence of a feedback loop: we found that mRNA levels of Smad5 do not change significantly on BMP4 treatment (Supplementary Figure S5B), whereas Smad5 protein decreases in cells treated with high doses of BMP4, thus showing an opposite trend compared with those of the miRNAs (Figure 6d). 
a

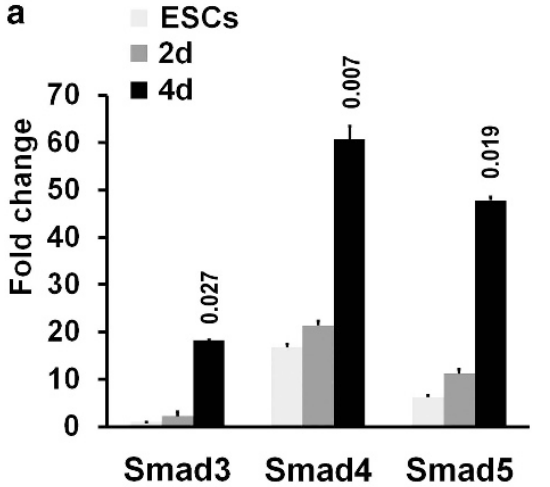

b
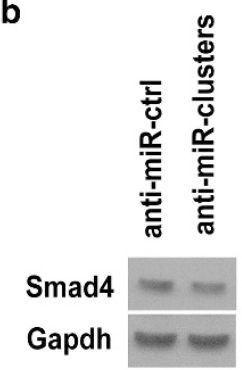

Smad5

C

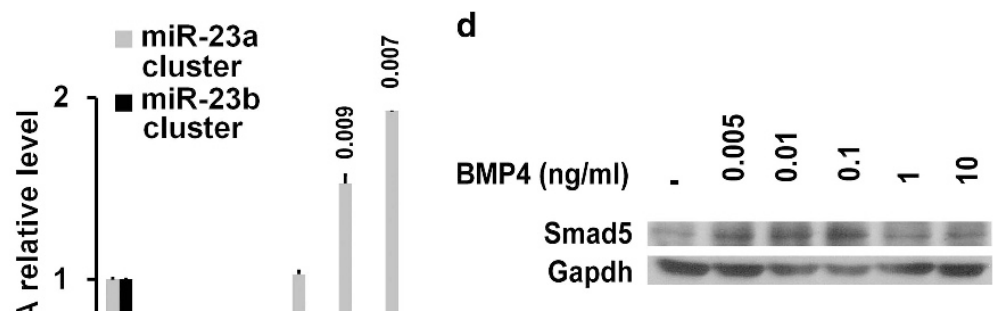

Figure 6 The miRNAs of the 23a and 23b clusters target Smad5. (a) The expression of the indicated Smads was analyzed by qPCR in ESCs and during neuroectodermal differentiation through SFEBs. Data are expressed as fold change over the level of the Smad3 mRNA in undifferentiated ESCs (fold change $=1$ ). (b) ESCs were transfected with the indicated anti-miRs and changes of Smad4 and 5 protein level were analyzed after $24 \mathrm{~h}$ by western blot. (c) ESCs were treated with the indicated doses of BMP4 and after $1 \mathrm{~h}$ the levels of the primary transcripts of miR-23a and miR-23b clusters were analyzed by qPCR. Data are expressed as fold change relative to the level of the miRNA cluster in untreated cells (fold change $=1$ ). The numbers over the bars indicate the $P$-Value. $(\mathbf{d})$ The protein level of Smad5 was analyzed in ESCs on treatment with the indicated doses of BMP4

\section{Discussion}

The study of the cytokines of the TGF $\beta$ superfamily, like BMP4 and Nodal/Activin, highlighted the very strong effects of these molecules on the in vitro naive state and on the differentiation of mouse ESCs. In particular, BMP4 represents an insurmountable barrier to the transition of ESCs into EpiSCs and then to the differentiation of EpiSCs into neuroectodermal precursors, thus favoring the choice of the mesendodermal fate. ${ }^{2}$ Therefore, it is quite expected that, in vivo, a fine tuning of the intracellular signaling downstream of BMP4 and of its crosstalk with other signaling pathways should exist to allow cells of the embryo to proceed through the various developmental stages. The results shown in this article uncover a new regulatory mechanism of the signaling downstream of BMP4 based on a small group of miRNAs encoded by two very similar miR-cluster genes. We observed that one of these cluster genes is under the control of BMP4 that activates its transcription, leading to the accumulation of miR-23a, 24 and 27a. This transcriptional activation is directly linked to BMP4 as demonstrated by the recruitment of phospo-Smad at the miR gene promoter. We have also observed that the miRs of this cluster target Smad5, which is expressed in ESCs; thus Smad5 and the miRs are engaged in a regulatory loop that modulates the response of the cells to BMP4 (Figure 7).
The tuning of the signal appears to be very accurate as indicated by the close relationship among the levels of the miRs (Figure 6c), the levels of Smad5 (Figure 6d) and the strength of the signal in terms of BMP4 concentrations at which ESCs are exposed to.

The mechanism described acts in parallel with a similar feedback loop we previously uncovered, based on miRs 125 a and 125b, which target the BMP4 co-receptor Dies1, and hence, also in this case, resulting in the downregulation of the signaling. ${ }^{19,31}$ Both mechanisms appear to be aimed at cushioning the intrinsically strong effects of BMP4, in agreement with the premise that the BMP4 signal, if not modulated, could be not compatible with the physiological progression of the differentiation events in vivo. However, the two mechanisms are not merely redundant, but they independently target two different events: the miR-125a-based loop affects the transition from ESCs to EpiSCs, which, on the contrary is unaffected by miR-23a, 24 and 27 a (Figures $4 a$ and b). The latter instead function by counteracting the apoptosis induced by BMP4. Indeed, it was previously demonstrated that low doses of BMP4 are able to induce the apoptosis of $\mathrm{ESCs},{ }^{29}$ and we demonstrated that suppression of the miRs of the 23a and 23b clusters leads to massive apoptosis of differentiating cells. Therefore, the results support the 


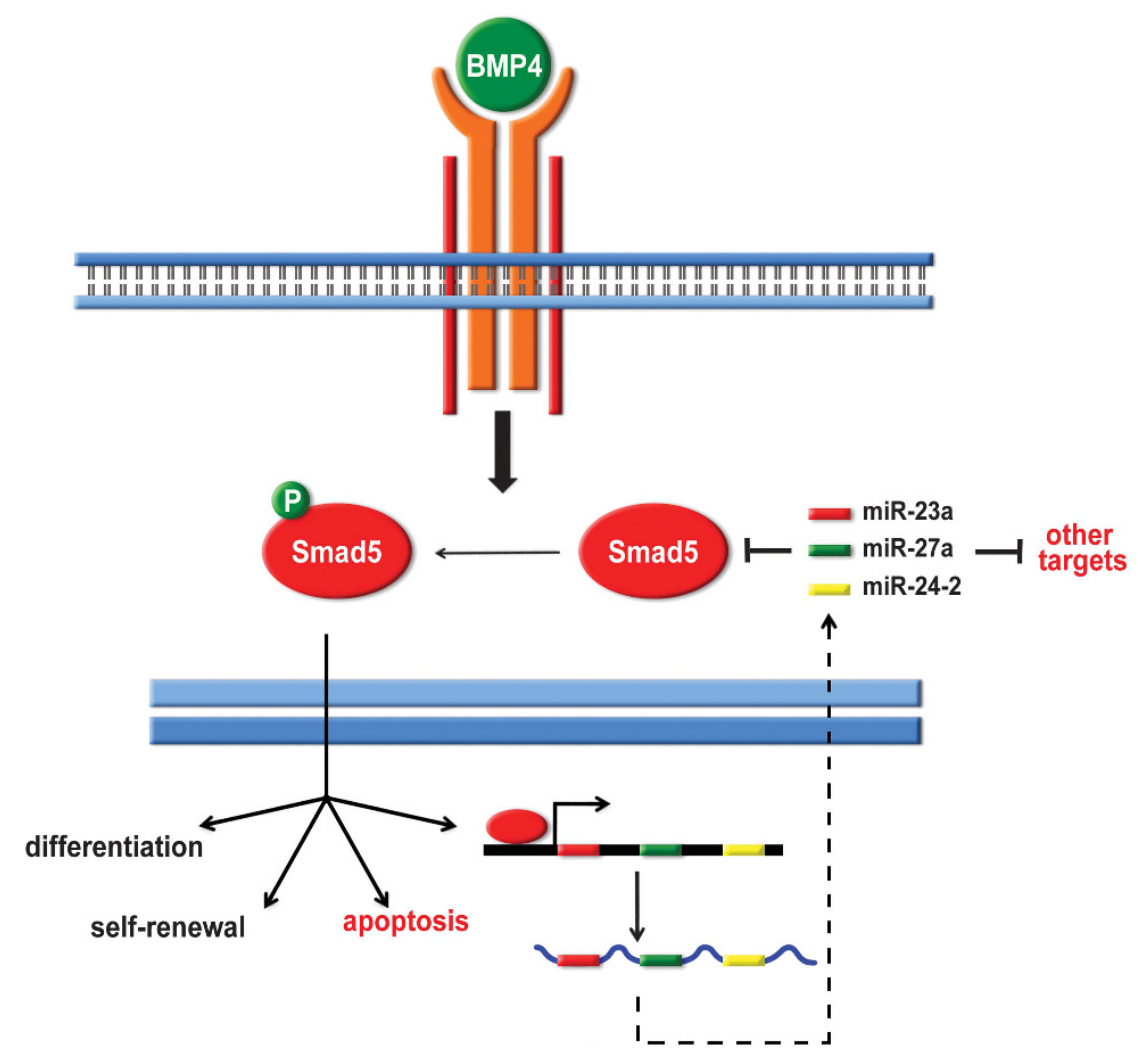

Figure 7 A feedback loop involves the BMP4 signaling pathway and the miRNAs of the 23a cluster. In ESCs, through the activation of Smads, BMP4 regulates many genes, thus affecting several functions, such as self-renewal, differentiation and apoptosis. BMP4 signaling also induces the transcription of the miRNAs of the 23a cluster, which in turn targets Smad5, thus downregulating the response of the cells to BMP4. As a consequence, these miRNAs protect differentiating ESCs from BMP4-dependent apoptosis. This effect could be dependent on the direct targeting by miRNAs of pro-apoptotic factors under the control of BMP4

possibility that the accumulation of these miRs is intended to protect ESCs from an inappropriate apoptosis induced by BMP4.

The anti-apoptotic role of these miRs was already described in other experimental systems ${ }^{32-34}$ and was also confirmed in our experiments where ESCs were exposed to genotoxic stress (Figure 4e). However, the mechanism through which the miRs exert this function is not clearly understood. We cannot exclude that there are other mRNAs targeted by these miRs whose downregulation could be responsible for their anti-apoptotic effects. Nevertheless, there is a close relationship between the targets of the miRs and BMP4 signaling, as the apoptosis induced by transfection of the anti-miRs is completely erased by the contemporary block of BMP4 signaling obtained by exposing the cells to Dorsomorphin (Figures 5b and c). Thus, it seems reasonable that there could be pro-apoptotic factors induced by BMP4 that may be targeted by miR-23a, 24 and $27 a$.

miR-23a/27a/24-2 is an intergenic miRNA cluster located on the chromosome 8 . These miRNAs belong to three different families and are not related in sequence. Therefore, their control by BMP4 and their targeting of at least one common mRNA (Smad5) suggests a coordinate action that the cells can adopt to modulate their fate. As mentioned above another cluster gene, located on the chromosome 13, generates a transcript containing three miRNAs of the same families, miR-23b/27b/24-1. Being identical (24-1 and 24-2) or very similar, apoptotic phenotype was clearly observed only when all the miRs of the two clusters were suppressed. Considering that BMP4 controls the transcription only of the cluster of miR-23a/27a/24-2, possible regulation of the cluster miR-23b/27b/24-1 by pathways different from that of BMP4 could be of interest. A similar dual control mechanism of BMP4 signaling was observed for the miRNAs of the 125 family, where miR-125a is directly regulated by $\mathrm{BMP} 4$, while miR-125b is not. ${ }^{19,31}$ Some miRNAs are regulated by BMP4 through a posttranscriptional mechanism, involving Smad proteins that engage miRNAs and promote their maturation by Drosha. ${ }^{35}$ miR-23a and 23b are among the miRs that are target of this mechanism, as they are induced in PASM cells on treatment with TGF $\beta$ or BMP4. This mechanism could be active in ESCs too, and, therefore, it could be responsible for the posttranscriptional accumulation of miR-23b.

In conclusion, in this study we have identified a group of miRNAs whose transcription is under the control of BMP4 signaling. These miRNAs, in turn, target Smad5, thus generating a feedback loop that modulates the response of the cells to this cytokine. Our results provide evidence that this molecular machinery controls apoptosis of ESCs during their differentiation thus contributing to the response of these cells to regulators of differentiation. 


\section{Materials and Methods}

Cell culture, transfection and treatment. E14Tg2a (BayGenomics, San Francisco, CA, USA) mouse ESCs and Sox1-GFP ESCs ${ }^{25}$ were maintained on feeder-free, gelatin-coated plates in the following medium: Glasgow Minimum Essential Medium (GMEM, Sigma, St. Louis, MO, USA) supplemented with $2 \mathrm{mM}$ glutamine, $1 \mathrm{mM}$ sodium pyruvate, $1 \mathrm{X}$ non-essential amino acids (all from Invitrogen, Carlsbad, CA, USA), $0.1 \mathrm{mM} \beta$-mercaptoethanol (Sigma), 10\% FBS (Hyclone Laboratories, Logan, UT, USA) and $10^{3} \mathrm{U} / \mathrm{ml}$ Leukemia Inhibitory Factor (LIF, Millipore, Billerica, MA, USA).

Transfection of plasmids and anti-miRs (Applied Biosystems, Foster City, CA, USA) was performed by plating $6 \times 10^{4} \mathrm{cell} / \mathrm{s} / \mathrm{cm}^{2} 16 \mathrm{~h}$ before transfection and using Lipofectamine2000 (Invitrogen) following the manufacturer's instructions.

For BMP4 treatment, ESCs were grown overnight in 10\% Knock-out Serum Replacement (KSR, Invitrogen) containing medium with LIF and then treated with BMP4 (R\&D Systems, Minneapolis, MN, USA) at the doses and for the time indicated.

For apoptosis induction, ESCs were irradiated with $5 \mathrm{~Gy}$ of $\mathrm{X}$ rays by using RS2000 Biological Irradiator (Rad Source) and incubated for $24 \mathrm{~h}$ before the analysis.

Differentiation of ESCs and generation of EpiSCs. ESC differentiation into neuroectoderm was induced though SFEB formation. ${ }^{2,20}$ SFEBs were induced by placing $1 \times 10^{6}$ ESCs in $100-\mathrm{mm}$ Petri dishes in the following differentiation medium: GMEM supplemented with $2 \mathrm{mM}$ glutamine, $1 \mathrm{mM}$ sodium pyruvate, $1 \times$ nonessential amino acids, $0.1 \mathrm{mM} \beta$-mercaptoethanol and $10 \% \mathrm{KSR}$.

Alternatively, the differentiation of Sox1-GFP ESCs ${ }^{25}$ into neural precursors was driven by plating $3 \times 10^{3}$ cells/cm on gelatin-coated dishes in differentiation medium as previously described. ${ }^{24}$

BMP4 (at the indicated doses) was added once in the differentiation medium when the cells were plated in Petri dishes to induce SFEB formation. Dorsomorphin (Sigma) was added at $2 \mu \mathrm{M}$ to differentiating cells and DMSO was used as negative control.

The formation of EpiSCs was induced adapting the methods of Hayashi et $\mathrm{al}^{22}{ }^{22}$ and Nakaki et al. ${ }^{23}$ In brief, ESCs were dissociated into a single-cell suspension with $0.05 \%$ Trypsin-EDTA at $37^{\circ} \mathrm{C}$ for $5 \mathrm{~min}$. Individual cells were then seeded in fibronectin-coated dishes at a density of $2.5 \times 10^{5} \mathrm{cell} / \mathrm{s} / \mathrm{cm}^{2}$ in ESC culture condition, and after $18 \mathrm{~h}$ the medium was switched to the following EpiSC medium: 1 vol of DMEM/F12 combined with 1 vol of Neurobasal medium, supplemented with $0.5 \%$ N2 supplement, $1 \%$ B27 supplement, 1\% KSR, $2 \mathrm{mM}$ glutamine (Invitrogen), $20 \mathrm{ng} / \mathrm{ml}$ Activin A (R\&D Systems), and $12 \mathrm{ng} / \mathrm{ml}$ bFGF (Invitrogen). Within 2 days in these conditions the cells undergo morphological transformation (including flattening, diminished cell-cell interactions and formation of cellular protrusions) and express epiblast markers.

RNA isolation, miRNA profiling and quantitative real-time PCR (qPCR). For quantitative PCR total RNA was extracted by using TRI-Reagent (Sigma). The first-strand CDNA was synthesized according to the manufacturer's instructions (M-MLV RT, New England BioLabs, Ipswich, MA, USA). qPCR was carried out with the 7500 Real-Time PCR System instrument and the Sequence Detection Systems (SDS) 1.4 software (Applied Biosystems) using Power SYBR Green PCR Master mix (Applied Biosystems). The housekeeping GAPDH mRNA was used as an internal standard for normalization, using $2^{-\Delta \Delta \mathrm{Ct}}$ method. Genespecific primers used for amplification are listed in Supplementary Table S2. For the analysis of single miRNA sequence specific LNA primers were used for the indicated miRNAs or U6 as internal control (both from Exiqon, Vedbaek, Denmark). For miRNA profiling, small RNA was isolated from ESCs treated or not with BMP4 with mirVana miRNA Isolation kit (Ambion, Austin, TX, USA). From each sample, $40 \mathrm{ng}$ of total RNA was used to synthesize single-stranded CDNA with Universal cDNA Synthesis Kit (Exiqon). Expression level of all miRNAs of Sanger miRBase v17 database was measured by using Mouse\&Rat Panel microRNA Ready-to-Use PCR (Exiqon) with the 7900HT instrument and the Sequence Detection Systems (SDS) v2.1 software (Applied Biosystems) using SYBR Green Master Mix (Exiqon). The miRNA profiling data were analyzed performing a comparative analysis by using the comparative $\mathrm{Ct}$ method $\left(2^{-\Delta \Delta \mathrm{Ct}}\right.$, RQManager 1.2 software; Applied Biosystems) using $\mathrm{U} 6$ as normalizer.

Antibodies and western blot analysis. Undifferentiated and differentiated ESCs were lysed in a buffer containing $1 \mathrm{mM}$ EDTA, $50 \mathrm{mM}$ Tris- $\mathrm{HCl}(\mathrm{pH} 7.5), 70$ $\mathrm{mMNaCl}, 1 \%$ Triton and protease inhibitor cocktail (Sigma), and analyzed by western blot. The following primary antibodies were used: rabbit Smad5 (1: 1000 Cell Signaling, Danvers, MA, USA), mouse Oct3/4 (1:2000 Santa Cruz Biotechnology, Santa Cruz, CA, USA), rabbit Nanog (1:1000 Calbiochem-EMD Biosciences, La Jolla, CA, USA) mouse GAPDH (1:1000 Santa Cruz
Biotechnology), goat Sox1 (1:100 Santa Cruz Biotechnology), rabbit Cleaved Caspase-3 (1: 1000 Cell Signaling), rabbit pro-Caspase-3 (1: 1000 Millipore), rabbit anti-Parp (1:400 Abcam), rabbit phospho-Smad1/5/8 (1:1000 Cell Signaling), rabbit Smad1 (1: 1000 Cell Signaling), rabbit Smad4 (1: 1000 GeneTex, Irvine, CA, USA). Antibody protein complexes were detected by HRP-conjugated antibodies and ECL (both from Amersham Pharmacia, Milan, Italy).

Chromatin immunoprecipitation (ChIP)-qPCR analysis. For ChIPqPCR analysis, ESCs were treated with $20 \mathrm{ng} / \mathrm{ml}$ BMP4 for $1 \mathrm{~h}$ and then were cross-linked with $1 \%$ formaldehyde for $10 \mathrm{~min}$ at room temperature and then with $125 \mathrm{mM}$ glycine. The chromatin was then sonicated to an average DNA fragment length of 500-1000 bp. Soluble chromatin extracts were immunoprecipitated using an anti-Phospho-Smad1,5,8 (Cell Signaling) antibody. Appropriate IgGs were used as negative control. Supernatant obtained without an antibody was used as an input control. After qPCR, the amount of precipitated DNA was calculated relatively to the total input chromatin and expressed as percentage of total chromatin or as fold enrichment relative to untreated samples. Oligonucleotide pairs are listed in Supplementary Table S1.

FACS analysis. Analysis of DNA content by propidium iodide incorporation was performed in permeabilized cells by flow cytometry. SFEBs at 4 days of differentiation were dissociated and $2 \times 10^{4}$ cells were collected, washed in PBS and resuspended in $200 \mu \mathrm{l}$ of a solution containing $0.1 \%$ sodium citrate $\mathrm{w} / \mathrm{v}, 0.1 \%$ Triton $\mathrm{X}-100 \mathrm{v} / \mathrm{v}$ and $50 \mu \mathrm{g} / \mathrm{ml}$ propidium iodide (Sigma Chemical, Perth, WA, Australia). After incubation at $4^{\circ} \mathrm{C}$ for $30 \mathrm{~min}$ in the dark, cell nuclei were analyzed with a FACScan flow cytometer (Becton Dickinson, Milan, Italy). Cellular debris was excluded from the analysis by raising the forward scatter threshold, and the DNA content of the nuclei was registered on a logarithmic scale. The percentage of the elements in the hypodiploid region was calculated.

Phosphatidylserine externalization was investigated by annexin $V$ staining. In brief, SFEBs at 4 days of differentiation were dissociated and $1 \times 10^{5}$ cells were collected and resuspended in $100 \mu \mathrm{l}$ of binding buffer (10 mM Hepes/NaOH pH 7.5, $140 \mathrm{mM}$ $\mathrm{NaCl}$, and $2.5 \mathrm{mM} \mathrm{CaCl}_{2}$ ) containing $5 \mu \mathrm{l}$ of annexin V-FITC (Pharmingen/Becton Dickinson, San Diego, CA, USA) for $15 \mathrm{~min}$ at room temperature in the dark. Then, $400 \mu$ l of the same buffer was added to each sample and the cells were analyzed with a Becton Dickinson FACScan flow cytometer.

Immunostaining, bromodeoxyuridine assay and microscopy. Undifferentiated ESCs and EpiSCs were fixed in 4\% paraformaldehyde and permeabilized with $0.2 \%$ Triton X-100 in 10\% FBS (Invitrogen)/ $1 \%$ BSA in 1 X PBS for $15^{\prime}$ at room temperature. Thus, the samples were incubated with primary antibodies and with an appropriate secondary antibody reported below. SFEBs were collected at the indicated differentiation day fixed in $4 \%$ paraformaldehyde and dehydrated with increasing percentages of ethanol. Samples were embedded in paraffin, sectioned in 7- $\mu \mathrm{m}$ slices and mounted on glass slides. After rehydration and permeabilization, the samples were treated as previously described. ${ }^{20}$ Nuclei were counterstained with DAPI (Calbiochem, $1: 1000$ ) or, for confocal analysis, with DRQ5 (Cell Signaling, $1: 1000)$ as indicated in the Figures. The following primary antibodies were used: anti-Oct3/4 (1:200), anti-Nanog (1:500; Calbiochem), antiSox1 $(1: 100)$ and anti-cleaved caspase $3(1: 300)$. Alexa Fluor 594 or 488 secondary antibodies were used (1:400; Invitrogen). For BrdU experiments, subconfluent ESCs were incubated in ESC medium containing BrdU for $1 \mathrm{~h}$, and then the cells were processed for immunofluorescence with BrdU labeling and detection kit (Roche, Basel, Switzerland). Images were captured with an inverted microscope (DMI4000; Leica Microsystems, Heidelberg, Germany) with Leica Application Suite Advanced Fluorescence (LAS AF) software (Leica Microsystems). Confocal microscopy was performed with a Leica TCSSMD FLIM microscope (Leica Microsystems) using LAS AF software (Leica Microsystems). When required, the brightness, contrast and color balance of the images were adjusted in Photoshop CS2 (Adobe Systems, San Jose, CA, USA).

Statistical analysis. All values represent the means \pm S.E. of at least three independent experiments. qPCR data are presented as fold change relative to the indicated reference sample. Whenever necessary, statistical significance of the data was analyzed using Student's $t$-test and the $P$-Value was reported in the figures.

\section{Conflict of Interest}

The authors declare no conflict of interest. 
Acknowledgements. This work was supported by grants from Associazione Italiana Ricerca sul Cancro and from Italian Ministry of University and Research for the projects: Futuro in Ricerca 2013 (RBFR13YZ2Y), PON 01 02782 and CESMA. A Vocca and A Gargiulo were supported by fellowships from Project STRAIN. We thank Danila Scarpa for the technical assistance and Dr Tiziana Parisi for the critical reading of the manuscript. The Sox1-GFP cells were kindly provided by Professor Austin Smith.

1. Tesar PJ, Chenoweth JG, Brook FA, Davies TJ, Evans EP, Mack DL et al. New cell lines from mouse epiblast share defining features with human embryonic stem cells. Nature 2007; 448: 196-199.

2. Zhang $\mathrm{K}$, Li L, Huang $\mathrm{C}$, Shen $\mathrm{C}$, Tan F, Xia $\mathrm{C}$ et al. Distinct functions of BMP4 during different stages of mouse ES cell neural commitment. Development 2010; 137: 2095-2105.

3. Ying QL, Nichols J, Chambers I, Smith A. BMP induction of Id proteins suppresses differentiation and sustains embryonic stem cell self-renewal in collaboration with STAT3. Cell 2003; 115: 281-292

4. Fei T, Xia K, Li Z, Zhou B, Zhu S, Chen H et al. Genome-wide mapping of SMAD target genes reveals the role of BMP signaling in embryonic stem cell fate determination. Genome Res 2010; 20: 36-44

5. Kawasaki H, Mizuseki K, Nishikawa S, Kaneko S, Kuwana Y, Nakanishi S et al. Induction of midbrain dopaminergic neurons from ES cells by stromal cell-derived inducing activity. Neuron 2000; 28: 31-40.

6. Tropepe V, Hitoshi S, Sirard C, Mak TW, Rossant J, van der Kooy D et al. Direct neural fate specification from embryonic stem cells: a primitive mammalian neural stem cell stage acquired through a default mechanism. Neuron 2001; 30: 65-78.

7. Gouon-Evans V, Boussemart L, Gadue P, Nierhoff D, Koehler Cl, Kubo A et al. BMP-4 is required for hepatic specification of mouse embryonic stem cell-derived definitive endoderm. Nat Biotechnol 2006; 24: 1402-1411.

8. Nostro M, Cheng X, Keller GM, Gadue P. Wnt, Activin and BMP signaling regulate distinct stages in the developmental pathway from embryonic stem cells to blood. Cell Stem Cell 2008; 2: 60-71

9. Parisi S, Russo T. Regulatory role of Klf5 in early mouse development and in embryonic stem cells. Vitam Horm 2011; 87: 381-397.

10. Yeo JC, Ng HH. The transcriptional regulation of pluripotency. Cell Res 2013; 23: 20-32.

11. Bernstein E, Kim SY, Carmell MA, Murchison EP, Alcorn H, Li MZ et al. Dicer is essential for mouse development. Nat Genet 2003; 35: 215-217.

12. Wang $Y$, Medvid R, Melton $C$, Jaenisch $R$, Blelloch $R$. DGCR8 is essential for microRNA biogenesis and silencing of embryonic stem cell self-renewal. Nat Genet 2007; 39: 380-385.

13. Houbaviy HB, Murray MF, Sharp PA. Embryonic stem cell-specific MicroRNAs. DevCell 2003; 5: 351-358

14. Suh MR, Lee Y, Kim JY, Kim SK, Moon SH, Lee JY et al. Human embryonic stem cells express a unique set of microRNAs. Dev Biol 2004; 270: 488-498.

15. Sinkkonen L, Hugenschmidt T, Berninger $P$, Gaidatzis D, Mohn F, Artus-Revel CG et al. MicroRNAs control de novo DNA methylation through regulation of transcriptional repressors in mouse embryonic stem cells. Nat Struct Mol Biol 2008; 15: 259-267.

16. Marson A, Levine SS, Cole MF, Frampton GM, Brambrink T, Johnstone S et al. Connecting microRNA genes to the core transcriptional regulatory circuitry of embryonic stem cells. Cell 2008; 134: 521-533.

17. Martinez NJ, Gregory RI. MicroRNA gene regulatory pathways in the establishment and maintenance of ESC identity. Cell Stem Cell 2010; 7: 31-35.
18. Tarantino C, Paolella G, Cozzuto L, Minopoli G, Pastore L, Parisi S et al. miRNA 34a, 100, and 137 modulate differentiation of mouse embryonic stem cells. FASEB J 2010; 24: 3255-3263.

19. Parisi S, Battista M, Musto A, Navarra A, Tarantino C, Russo T et al. A regulatory loop involving Dies1 and miR-125a controls BMP4 signaling in mouse embryonic stem cells. FASEB J 2012; 26: 3957-3968.

20. Aloia L, Parisi S, Fusco L, Pastore L, Russo T. Differentiation of embryonic stem cells 1 (Dies1) is a component of bone morphogenetic protein 4 (BMP4) signaling pathway required for proper differentiation of mouse embryonic stem cells. J Biol Chem 2010; 285 : 7776-7783.

21. Ying Q. L, Nichols J, Chambers I, Smith A. BMP induction of Id proteins suppresses differentiation and sustains embryonic stem cell self-renewal in collaboration with STAT3. Cell 2003; 115: 281-292.

22. Hayashi K, Ohta H, Kurimoto K, Aramaki S, Saitou M. Reconstitution of the mouse germ cell specification pathway in culture by pluripotent stem cells. Cell 2011; 146: 519-532.

23. Nakaki F, Hayashi K, Ohta H, Kurimoto K, Yabuta Y, Saitou M et al. Induction of mouse germcell fate by transcription factors in vitro. DNA repair in murine embryonic stem cells and differentiated cells. Nature 2013; 501: 222-226.

24. Parisi S, Tarantino C, Paolella G, Russo T. A Flexible Method to Study Neuronal Differentiation of Mouse Embryonic Stem Cells. Neurochem Res 2010; 35: 2218-2225.

25. Ying QL, Stavridis M, Griffiths D, Li M, Smith A. Conversion of embryonic stem cells into neuroectodermal precursors in adherent monoculture. Nat Biotechnol. 2003; 21: 183-186.

26. Tichy ED, Stambrook PJ. DNA repair in murine embryonic stem cells and differentiated cells. Exp Cell Res 2008; 314: 1929-1936.

27. Zheng GX, Ravi A, Calabrese JM, Medeiros LA, Kirak O, Dennis LM et al. A latent prosurvival function for the mir-290-295 cluster in mouse embryonic stem cells. PLoS Genet. 2011; 7: e1002054

28. Tichy ED, Stephan ZA, Osterburg A, Noel G, Stambrook PJ. Mouse embryonic stem cells undergo Charontosis, a novel programmed cell death pathway dependent upon cathepsins, p53, and EndoG, in response to etoposide treatment Elisia D. Stem Cell Res 2013; 10: 428-441.

29. Gambaro K, Aberdam E, Virolle T, Aberdam D, Rouleau M. BMP-4 induces a Smaddependent apoptotic cell death of mouse embryonic stem cell- derived neural precursors. Cell Death Differ 2006; 13: 1075-1087.

30. Rogler CE, Levoci L, Ader T, Massimi A, Tchaikovskaya T, Norel R et al. MicroRNA-23b cluster microRNAs regulate transforming growth factor-beta/bone morphogenetic protein signaling and liver stem cell differentiation by targeting Smads. Hepatology 2009; 50 : 575-584.

31. Battista M, Musto A, Navarra A, Minopoli G, Russo T, Parisi S et al. miR-125b Regulates the Early Steps of ESC Differentiation through Dies 1 in a TGF-Independent Manner. Int J Mol Sci 2013; 14: 13482-13496.

32. Chen $Q$, Xu J, Li L, Li H, Mao S, Zhang $F$ et al. MicroRNA-23a/b and microRNA-27a/b suppress Apaf-1 protein and alleviate hypoxia-induced neuronal apoptosis. Cell Death Dis 2014; 5: e1132.

33. Huang S, He X, Ding J, Liang L, Zhao Y, Zhang Z et al. Upregulation of miR-23a approximately 27 a approximately 24 decreases transforming growth factor-beta-induced tumor-suppressive activities in human hepatocellular carcinoma cells. Int J Cancer 2008; 123: $972-978$.

34. Nguyen T, Rich A, Dahl R. MiR-24 promotes the survival of hematopoietic cells. PLoS One 2013; 8: e55406.

35. Davis BN, Hilyard AC, Nguyen PH, Lagna G, Hata A. Smad proteins bind a conserved RNA sequence to promote microRNA maturation by Drosha. Mol Cell 2010; 39: 373-384.

\section{Supplementary Information accompanies this paper on Cell Death and Differentiation website (http://www.nature.com/cdd)}

